\title{
Generation and measurement of multi megagauss fields in inertial magnets
}

\author{
N NALINI, T C KAUSHIK and S C GUPTA \\ Applied Physics Division, Bhabha Atomic Research Centre, Mumbai 400085 \\ e-mail: nalini@barc.gov.in
}

MS received 5 March 2010; revised 5 August 2010; accepted 13 September 2010

\begin{abstract}
We present here the development of a facility to generate high (multi megagauss) magnetic field of 4 to $5 \mu$ s rise time, using inertial magnets. The facility includes a low inductance, high current capacitor bank $(280 \mathrm{~kJ} / 40 \mathrm{kV})$ and an inertial magnet, which is a copper disk machined to have a keyhole in it. As the high current from the capacitor bank is discharged through the copper disk, a high magnetic field is produced along its axis, before it is destroyed by the combined effect of the dynamic loading and skin effect. A maximum peak magnetic field of $257 \mathrm{~T}$ is realized, when the magnet with $3.6 \mathrm{~mm}$ inner diameter, $35 \mathrm{~mm}$ outer diameter and $5 \mathrm{~mm}$ length, is powered by the capacitor bank charged to $28 \mathrm{kV}$ $(134 \mathrm{~kJ})$. The transient magnetic field is measured using a B dot probe with an error of $\pm 25 \mathrm{~T}$. The probe in most of high field shots $(>200 \mathrm{~T})$ got destroyed before recording the peak field and the trailing edge of the magnetic field. Experimental evidence of enhancement of the probe survival for longer time in copper disks using spatial non-uniform conductivity with $1 \mathrm{~mm}$ thick SS brazed to the inner wall of the inertial magnet is also reported.
\end{abstract}

Keywords. Megagauss; transient magnetic field; single turn coil technique; B dot probes.

\section{Introduction}

Generation of multi-mega gauss (MG) magnetic fields using inertial magnets became possible with the use of low inductance capacitive energy storage devices. Inertial magnets are single turn solenoids where the high current flowing through it produces a high magnetic field at its axis. Magnets carrying mega ampere currents generate mega gauss (MG) magnetic fields $(1 \mathrm{MG}=100 \mathrm{~T})$. The high magnetic field could persist as long as the magnet preserved its electrical identity. The combined effect of high magnetic pressure associated with high magnetic field (4 GPa corresponds to $100 \mathrm{~T}$ ) and heating due to high magnetic field diffusion into the magnets results in violent explosion of the metal wall of the magnet confining the fields. The metal wall is pushed away from the magnetic field at the rate of several mm per $\mu \mathrm{s}$. The coil destruction can be delayed with the decreased current pulse duration, necessitating a fast, low inductance, high energy capacitor bank driving source. 
This is a matured technology lasting for more than 50 years, since its first demonstration by Furth \& Waniek (1956), followed by a detailed work describing the inertial effects by Furth et al (1957). Knoepfel (1970) and Miura \& Herlach (1985) provide detail information on the generation, limiting factors and measurement of pulsed high magnetic fields. Several works on high magnetic fields using inertial magnet have been conducted following this. But most of these experiments used inertial magnets with $2-3 \mathrm{~mm}$ wall thickness made out of copper sheets. The thinner magnets are mainly chosen because of the limitations in the available energy. Using thin walled magnets the highest field is reported by Portugall et al (1999) generation of $311 \mathrm{~T}$ using single turn coil with $5 \mathrm{~mm}$ inner diameter, $5 \mathrm{~mm}$ length and $3 \mathrm{~mm}$ thickness powered by $200 \mathrm{~kJ}$ capacitor bank energy. Recently, a $300 \mathrm{~T}$ microsecond duration pulsed field generating facility has been reported for structural phase transition study on Plutonium at NHMFL, Los Alamos National Laboratory by Singleton (2004). The only reported work on heavy inertial magnets is by Shearer (1969), aimed to study the interaction of the high magnetic field with magnets inner wall. The thick and heavy magnets suffer large deformations compared to the relatively thinner coils. The extent of deformation suffered by heavy inertial magnets of $1 \mathrm{~mm}$ inner diameter powered by $820 \mathrm{~kJ}$ capacitor bank generating a peak magnetic field of $355 \mathrm{~T}$ peak field is shown by Shearer (1969).

The current source commonly used with thinner magnets in all the reported experiments has a rise time of less than $2 \mu \mathrm{s}$. The current source we intend to use, to power our magnet has a peak current rise time of $4 \mu \mathrm{s}$. Usage of similar thinner coils with the kind of source like ours would result in faster expansion and poor energy transfer. The dimension of the magnet is so chosen that it undergoes minimum deformation before the current peak. Our inertial magnet is neither so massive like that of Shearer (1969), nor is thin-walled as in other facilities. Peak field record loss due to the premature damage to the probe similar to that of Shearer (1969) has been encountered in some of our high field shots greater than $200 \mathrm{~T}$. A novel method of a layer of SS brazed along the inner wall of the copper magnet has been effective in preventing such premature damage to the probe.

In this article, we will first describe the technical implementation of the mega gauss generating system and diagnostics in section 2 . The experimental results along with the discussions are provided in section 3 , followed by the conclusion in section 4 .

\section{Mega gauss generating facility}

We have installed a high energy $(280 \mathrm{~kJ})$, low inductance $(22 \mathrm{nH})$ capacitor bank capable of delivering an oscillating electrical discharge of $4 \mathrm{MA}$ peak current for a charging voltage of $40 \mathrm{kV}$. This source with co-axial collector plate assembly has been used for the pulsed magnetic field generating loads like inertial magnets and imploding liners. The capacitor bank, associated sub systems, inertial magnet, current and magnetic field diagnostics are discussed in the following.

\subsection{High current source}

Figure 1 shows the schematic of our $280 \mathrm{~kJ} / 40 \mathrm{kV}$ capacitor bank named MAGIC (Mega Ampere Generator for Intense Compression). The main components of this source are the energy storage capacitors each mounted with its own spark gap. There are 48 numbers of $7 \cdot 1 \mu \mathrm{F} / 40 \mathrm{kV}$ capacitors, all connected in parallel to form the $341 \mu \mathrm{F} / 40 \mathrm{kV}$ capacitor bank. Parallel connection of capacitors provides higher capacitance and lower inductance, which results in high current output. Each capacitor has an in-built four electrode type pressurized 


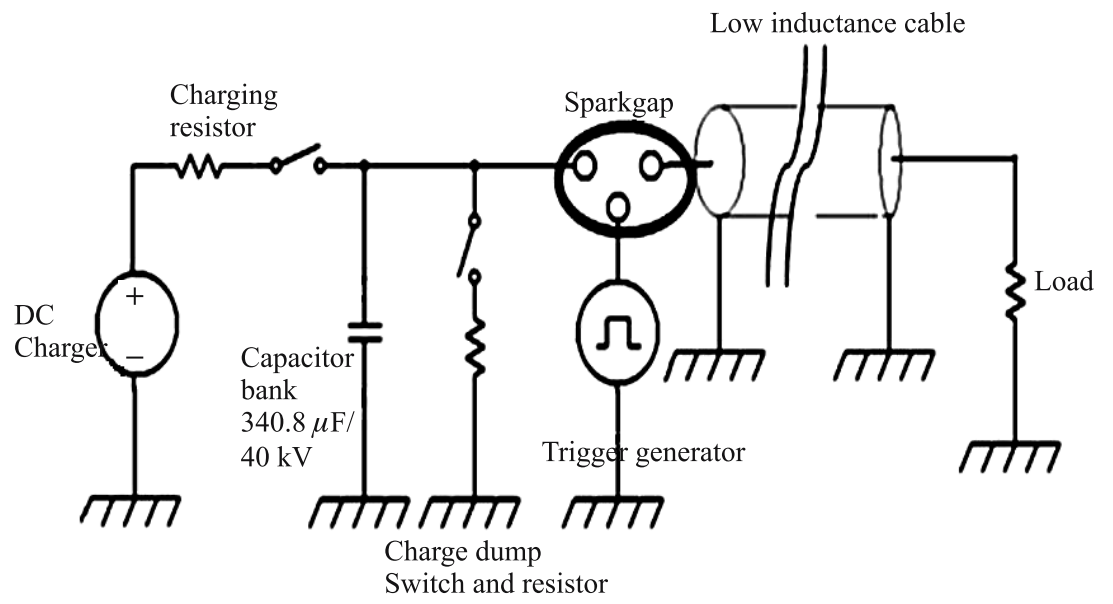

Figure 1. High current source schematics.

spark gap that works on field distortion principle. Owing to its four electrode geometry, the spark gaps offer a low jitter (a few ns) and negligible inductance (a few $\mathrm{nH}$ ).

The 48 numbers of trigger pulse required to trigger all the capacitors simultaneously should have minimum jitter $(<10 \mathrm{~ns})$, higher voltage $(40 \mathrm{kV})$, higher time derivate of voltage $(4 \mathrm{kV} / \mathrm{ns})$ and moderate energy $(2 \mathrm{~J})$. Figure 2 shows the circuit diagram of the trigger pulse

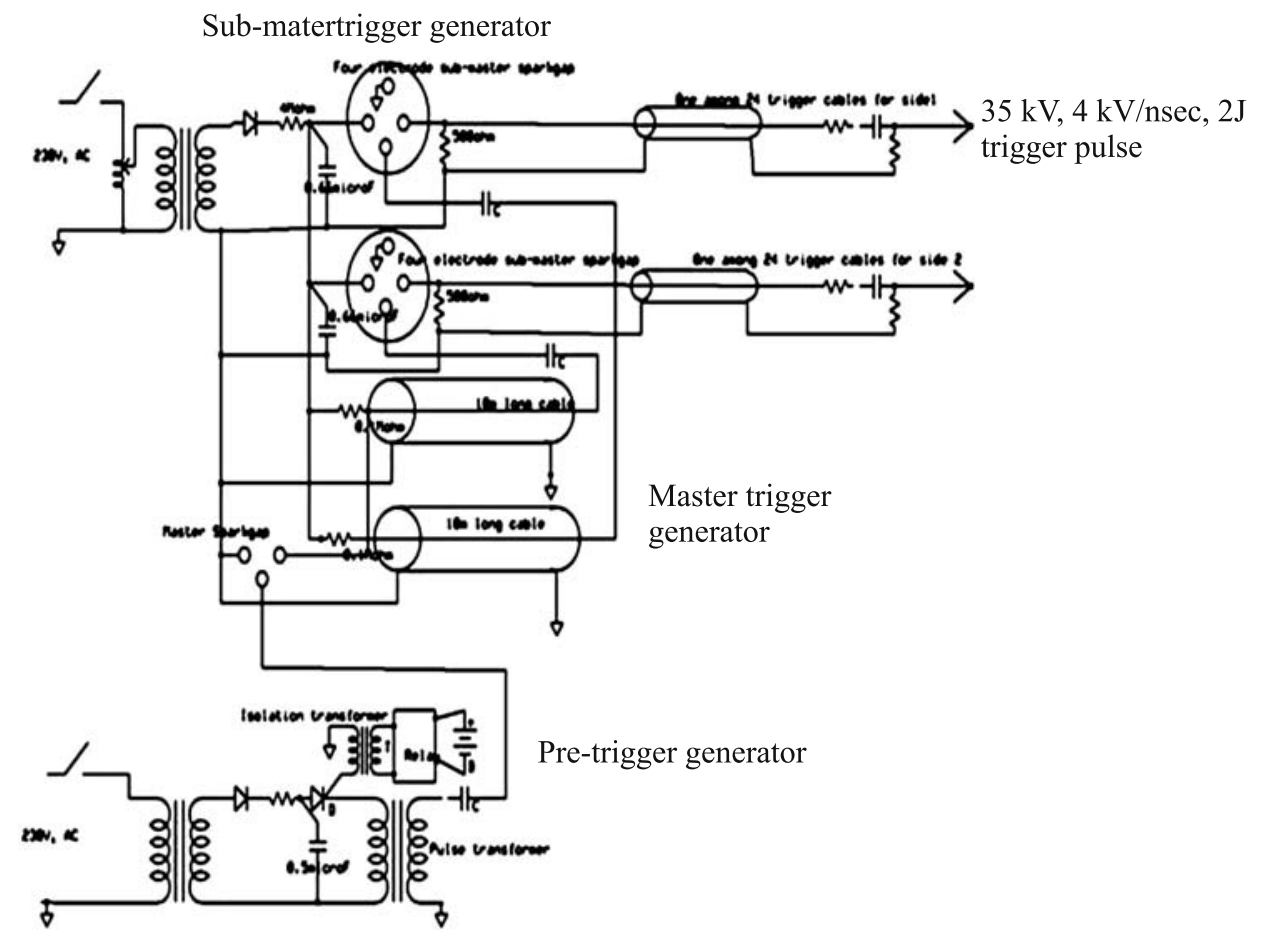

Figure 2. Circuit for generating 48 numbers of trigger pulse simultaneously. 
generator. Three stages are used to generate pulses of desired characteristics mentioned above. The first stage is a pre-trigger generator, the second stage is the master trigger generator and the third is the sub-master trigger generator. The pre-trigger generator consists of a $0.5 \mu \mathrm{F}$, $300 \mathrm{~V}$ capacitor connected through a relay to the input of the ferrite core pulse transformer. When the relay is closed by a 'command' TTL pulse from the control panel, the pulse transformer generates a $30 \mathrm{kV}, 1 \mu$ s rise time $20 \mathrm{~mJ}$ pulse at its output. The master trigger generator includes two RG 164 cables as the energy storage elements. Each cable is $10 \mathrm{~m}$ long, so as to have equal delay and pulse width of $100 \mathrm{~ns}$. These cables which act as pulse forming lines are initially charged to a voltage of $35 \mathrm{kV}$. These lines are discharged through the load by closing master spark gap using trigger pulse output of pre-trigger generator. The output pulses of master trigger generator are two numbers of $35 \mathrm{kV}$ pulses with $100 \mathrm{~ns}$ rise time. The sub-master trigger generator includes two parallel connections of $0.66 \mu \mathrm{F}$ capacitors charged to $35 \mathrm{kV}$, triggered using a low inductance four electrode spark gap. The energy of each pulse produced by the sub-master trigger generator is greater than $2 \mathrm{~J}$, which is more than sufficient to trigger our capacitor bank spark gaps. A common constant current power supply $30 \mathrm{kV}, 10 \mathrm{~mA}$ is used to charge the sub-master trigger generator capacitors and the master trigger generator cables. This trigger generator delivers 48 numbers of $35 \mathrm{kV}, 4 \mathrm{kV} / \mathrm{ns}$, and $2 \mathrm{~J}$ trigger pulses and ensures simultaneous trigger of all the 48 capacitors with switching jitter less than $30 \mathrm{~ns}$.

The capacitors are connected to a collector plate by 192 low inductance co-axial cables. The collector plate assembly consists of two SS plates electrically insulated and mechanically held together. The energy output of MAGIC would be delivered to load connected to it in a damped ringing electrical discharge. Two water resistors of $750 \Omega$ have been used as charging resistor and as dump for entire capacitor bank energy. The collector plate and the load assembly are enclosed by the vacuum chamber that can be evacuated to less than $10^{-5} \mathrm{mbar}$.

\subsection{Inertial magnet}

The Mega gauss generating system includes inertial magnet as the load, connected across the collector plate. When the high current output of the capacitor bank is discharged through the inertial magnet, it generates a field at its centre proportional to the initial energy stored in the capacitor. Figure 3 shows the inertial magnet geometry. To comply with the fact that single turn coil with inner diameter comparable to or smaller than the length generate maximum

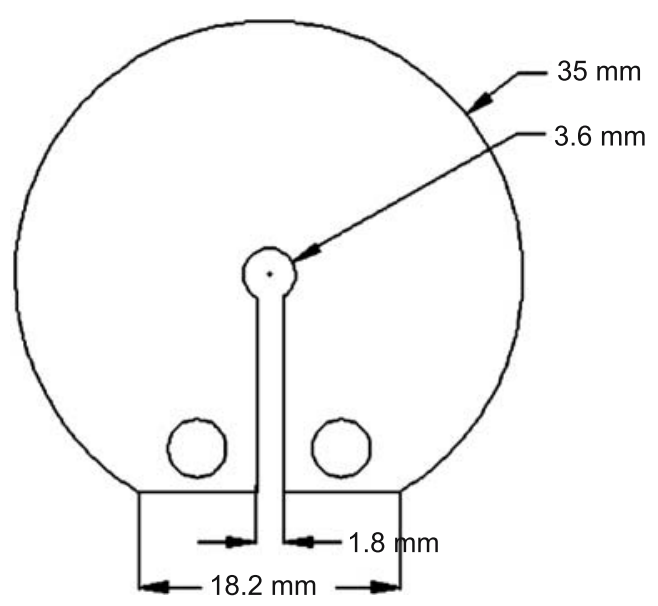

Figure 3. Inertial magnet geometry. 


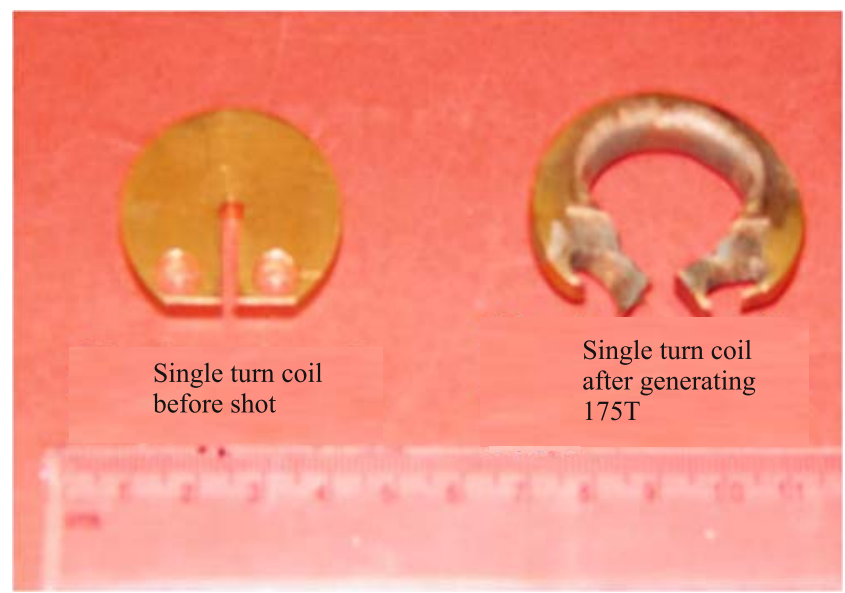

Figure 4. Single turn coil before and after generating a peak field of $175 \mathrm{~T}$.

peak magnetic field Shneerson \& Krivosheev (2004), the inner diameter is chosen as $3.6 \mathrm{~mm}$ diameter and length $5 \mathrm{~mm}$. This magnet is first bolted to the SS vertical connections which are in turn connected to the co-axial collector plates. The vertical SS connections are damaged in all high field shots along with the coil. Four layers of $17 \mu \mathrm{m}$ thick Mylar sheets are used for insulating the slot in the coil. Figure 4 shows the inertial magnet before and after generating peak magnetic field strength of $175 \mathrm{~T}$. The associated high magnetic pressure and the heating due to the high magnetic field diffusion results in plastic deformation of the magnet along the skin layer and material displacement in radial and axial directions. This accounts for the increase in the inner radius of the magnet after shot much greater than the initial one (figure 4).

\subsection{B dot probes}

The MG field results in high radial stress proportional to the square of the peak magnetic field generated $\left(\mathrm{B}^{2} / 2 \mu_{0}\right)$. The yield strength of copper $(200 \mathrm{MPa})$ limits its capability to generate a static field of $20 \mathrm{~T}$ non-destructively. This destructive and transient nature of the field and the space limitations pose great problems in high magnetic field measurements. The $\mathrm{B}$ dot probe used here is basically a small solenoid with two turns and an effective area of $3 \cdot 14 \times 10^{-6} \mathrm{~m}^{2}$. When it is placed in a varying magnetic field, there is an induced voltage across it, which is numerically integrated to get the magnetic field output. B dot probes generally need to be replaced in every shot, necessitating proper calibration of every probe before the actual experiments.

A $1 \mu \mathrm{F} / 8 \mathrm{kV}$ capacitor bank delivering $3 \mu \mathrm{sec}$ rise time current pulse to a standard solenoid is used to calibrate our Bdot probe. The Bdot probe is kept at the centre of the solenoid. The theoretical centre axial field strength is calculated by

$$
B_{Z}(0,0)=\frac{\mu_{0} N I}{l} K_{H}
$$

where, $N$ is the number of turns, $I$ is the current input, $l$ is the length of the coil, $K_{H}$ is the geometric factor (Knoepfel 1970), for our standard solenoid it is equal to 0.87. The accuracy of theoretical calculation relies completely on the accuracy with which the current input to solenoid is measured. The current through solenoid is measured using Tektronix P6021 probe 
along with CT-5 high current transformer with an accuracy of $\pm 7 \%$. At $4 \mathrm{kA}$ current input to the solenoid, the theoretically calculated field at the centre of the solenoid is $1.203 \mathrm{~T}$. The voltage across the Bdot probe is numerically integrated to get a peak magnetic field of 1.244 T. The cumulative error in the magnetic field measurement using the B dot probe is $\pm 10 \%$. For the actual shot, the same Bdot probe placed at the centre of the single turn coil. The voltage across the Bdot probe amounts to a peak magnetic field of $257 \mathrm{~T}$ at the coil centre. The actual peak magnetic field of $257 \mathrm{~T}$ is measured with the Bdot probe with an error of $\pm 25 \mathrm{~T}$. An error of $\pm 10 \%$ in the high magnetic field measurements is very common in such electronically noisy environments using these inductive pick-up probes. Only, optical technique like Faraday probe can lead to high magnetic field measurements more close to the actual.

\subsection{Current probe}

The current through the inertial magnet is measured using an inductive pick up coil placed beneath the collector plate. The coil sensor is made using a 100 turns of flexible insulated wire wound over the insulation of the center core of the RG 58 cable with shielding removed. The coil is connected between the center core and the shielding. The induced voltage across the coil is fed through long shielded cable to the oscilloscope in the Faraday cage. Mega-ampere currents result in about hundreds of voltage induced across the sensor coil (for $1 \mathrm{MA}$ about $250 \mathrm{~V}$ ). This voltage is attenuated using an electronic attenuator before connecting it to the oscilloscope. A 4 channel oscilloscope with $500 \mathrm{MHz}$ bandwidth (Tektronix TDS 754D) is used for the signal display. The induced voltage is further integrated using a passive RC integrator with time constant of $116 \mu \mathrm{s}$. The integrator is put in an aluminium shielding enclosure and attached to the oscilloscope directly.

\section{Experimental results and discussion}

Among the series of experiments carried out with our inertial magnets, maximum central axial field has been obtained with copper disk of $3.6 \mathrm{~mm}$ inner diameter, $17.5 \mathrm{~mm}$ wall thickness and $5 \mathrm{~mm}$ length, with capacitor bank charged to $28 \mathrm{kV}(134 \mathrm{~kJ})$. The maximum rate of change of the magnetic field strength realized is $1.7 \times 10^{8} \mathrm{~T} / \mathrm{s}$ and the peak magnetic field recorded is $257 \mathrm{~T}$ at $2 \cdot 16 \mu \mathrm{sec}$ (figure 5). The probe has got destroyed earlier rendering the full field pulse unrecorded. This problem has occurred only in high field shots above $200 \mathrm{~T}$, similar magnetic field record loss has been reported by Shearer (1969). Figure 6 shows the results of experiment with copper disk of $5 \mathrm{~mm}$ inner diameter, $15 \mathrm{~mm}$ wall thickness and $10 \mathrm{~mm}$ length powered by the capacitor bank charged to $26 \mathrm{kV}$, in which the probe has successfully recorded the rising and the trailing edge of the magnetic field pulse. Peak field recorded is $197 \mathrm{~T}$.

Experiments are done with different magnets having different inner diameter and length with fixed outer diameter and with fixed source parameters of capacitor bank charged to $28 \mathrm{kV}$. Figure 7 shows the comparison between peak magnetic field generated and the ratio of coil inner diameter $(I D)$ to length $(l)$. Magnets with $I D / l$ of 0.7 to 1 result in maximum peak magnetic field strength. Magnets with $I D / l>1$, become comparable to exploding foil and experiences high expansion rate and poor energy transfer, therefore lead to faster magnetic field rise time but lesser peak field. Whereas magnets with increased length $I D / l<1$, lead to reduced peak magnetic field (equation 1) and wider magnetic field pulse, owing to its large inductance. With the outer diameter and length fixed, magnets with lesser inner diameter generates higher peak magnetic field. The probe for magnetic field diagnostics placed at the centre of the coil used in the experiments reported here generally has an outer 


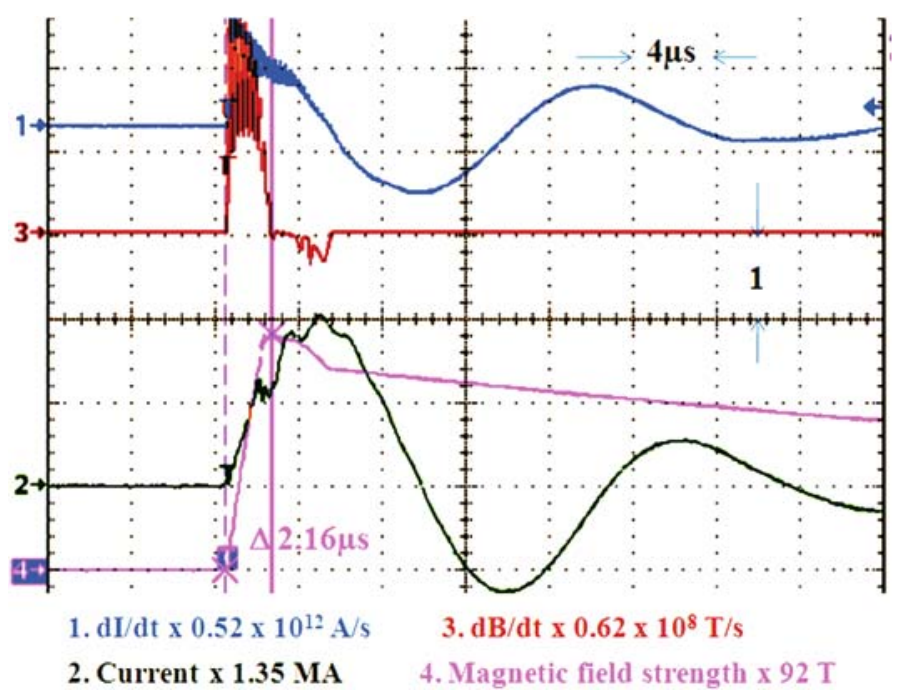

Figure 5. Scope record of the current through the magnet and the field generated and its time rate of change of an inertial magnet with $3.6 \mathrm{~mm}$ inner diameter, $5 \mathrm{~mm}$ long and $35 \mathrm{~mm}$ outer diameter.

diameter of 3 to $4 \mathrm{~mm}$. This limits the inner diameter of our magnet to $3.6 \mathrm{~mm}$, to comfortably accommodate the probe at the centre. However, when the coil used has an inner diameter close to the probe of outer diameter, there is a high probability of probe getting destroyed by the coil inner wall explosion as explained below.

The interaction of magnetic field generated by the magnet with the current carrying layer (Lorentz force $J \times B$ ) results in stress on the coil inner wall proportional to $B^{2}$ and the Joule heating in the coil is proportional to $J^{2}$, where $B$ is the field generated by the coil and $J$ is the current density. The coil inner wall undergoes a hydrodynamic compression,

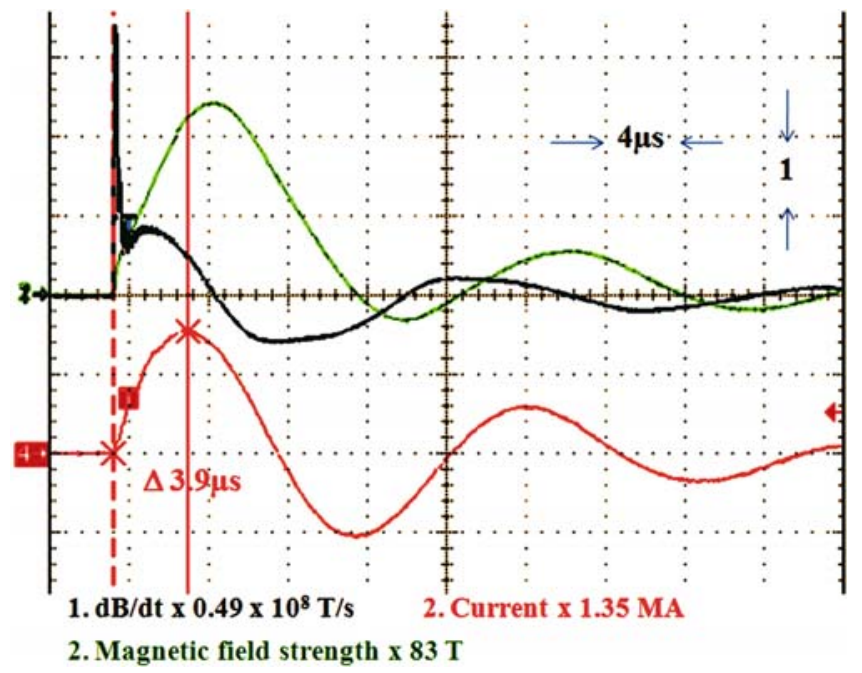

Figure 6. Scope record of the current through the magnet and the field generated and its time rate of change of an inertial magnet with $5 \mathrm{~mm}$ inner diameter, $10 \mathrm{~mm}$ long and $35 \mathrm{~mm}$ outer diameter. 


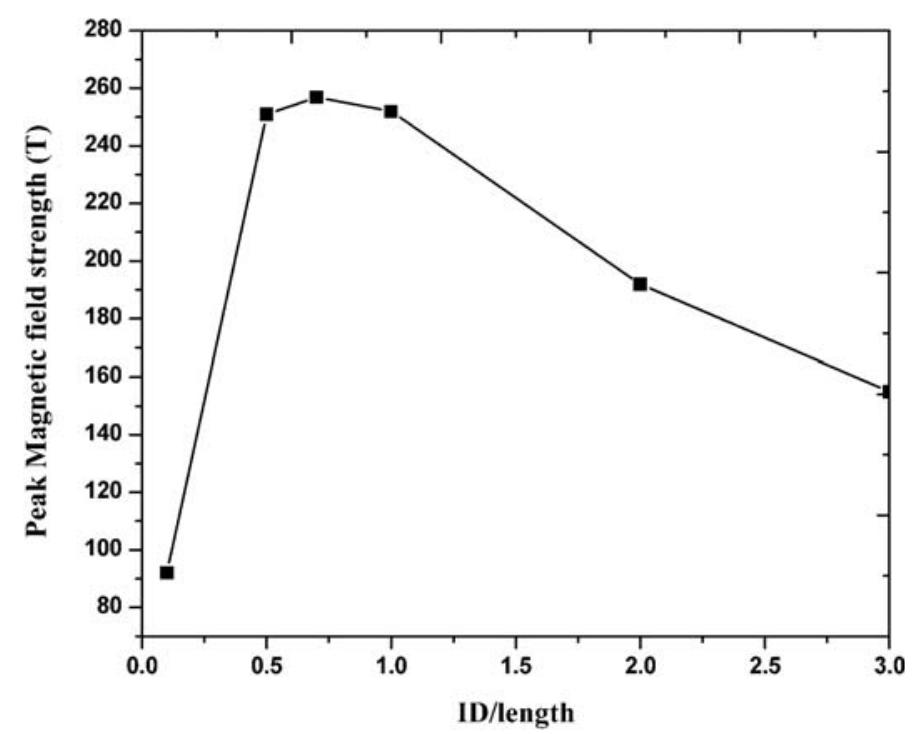

Figure 7. Peak magnetic field related to the magnets inner diameter to length ratio.

magnetic field diffuse into this heated and the compressed zone. This non-linear diffusion results in further heating of the zone and sudden transformation to vapor phase occurs. A low density; high temperature cloud of vaporized surface material expands into the high field volume as shown by Shearer (1969). This interacts with the probe to destroy it. It is also worth to note that higher is the capacitor bank charging voltage, lower is the time at which the probe is damaged, because the energy is transferred to the magnet at higher rate. It is expected that properly armored probe can be protected from such destruction. The inner diameter of the magnet is $3.6 \mathrm{~mm}$ and outer diameter of the probe is $3 \mathrm{~mm}$ with bare minimum space for any kind of armor glass or plastic tube to be put around probe for protection.

The other alternative thought about is to use a magnet with high tensile strength like SS. But materials with high tensile strength possess lower conductivity. Materials with lower conductivity have low flux trapping ability, necessitating a combination of materials to get the best performance. The general rule of mixtures for mechanical properties and current carrying capability are given by Frings \& Bockstal (1995). With combination coil made of copper and SS

$$
\begin{gathered}
\sigma_{A B}=\lambda \sigma_{A}+(1-\lambda) \sigma_{B}, \\
\Gamma_{A B}=\lambda \Gamma_{A}+(1-\lambda) \Gamma_{B},
\end{gathered}
$$

where $\lambda$ is the fraction of the material A (SS), $\sigma_{A B}, \sigma_{A}$, and $\sigma_{B}$ are the tensile strength of the combined material (AB), copper (A) and SS (B), $\Gamma_{A B}, \Gamma_{A}$, and $\Gamma_{B}$ are the current carrying capability of the combined material, copper (A) and SS (B). Tensile strength and current carrying capability of the hard copper are $390 \mathrm{Mpa}$ and $27 \times 10^{7} \mathrm{~A} \cdot \mathrm{s}^{1 / 2} \mathrm{~m}^{-2}$ and that of SS $2000 \mathrm{Mpa}$ and $3.5 \times 10^{7} \mathrm{~A} . \mathrm{s}^{1 / 2} \mathrm{~m}^{-2}$ (Frings \& Bockstal 1995). Copper coil with $1 \mathrm{~mm}$ SS lining will have approximately a diffusion time constant of $300 \mu \mathrm{sec}$ and net tensile strength of $490 \mathrm{Mpa}$. Introduction of $1 \mathrm{~mm} \mathrm{SS} \mathrm{lining} \mathrm{increases} \mathrm{the} \mathrm{tensile} \mathrm{strength} \mathrm{by} 26 \%$. Figure 8 


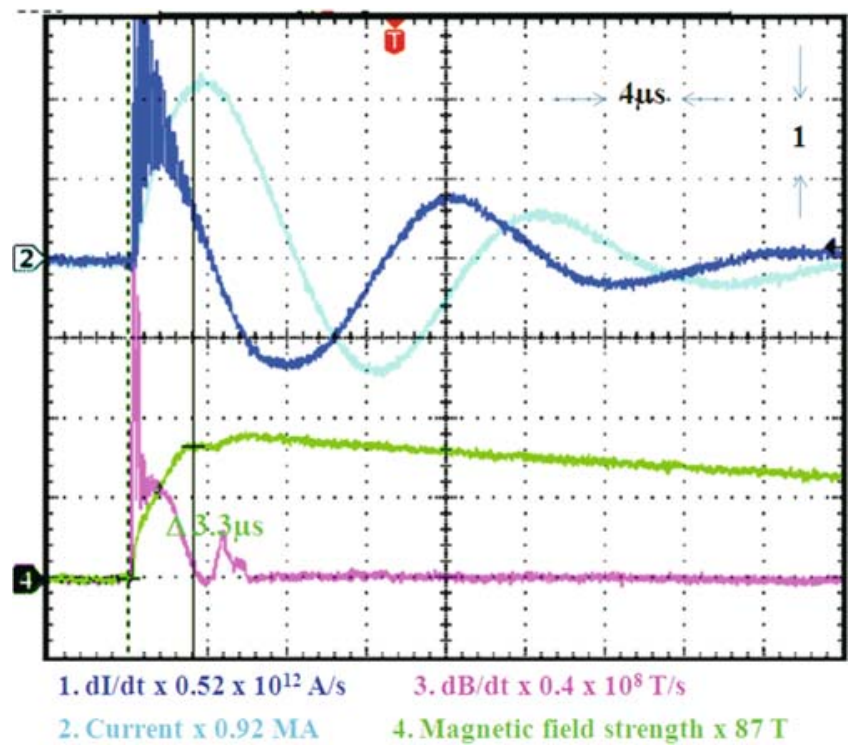

Figure 8. Experimental performance of an inertial magnet with $3.6 \mathrm{~mm}$ inner diameter, $3 \mathrm{~mm}$ long and $35 \mathrm{~mm}$ outer diameter without SS lining.

shows the rate of change of the current, time history of the current, rate of change of flux density and magnetic field strength time history of copper coil $3.6 \mathrm{~mm}$ inner diameter, $3 \mathrm{~mm}$ length and $35 \mathrm{~mm}$ outer diameter powered by the high current source charged to $20 \mathrm{kV}(68 \mathrm{~kJ})$, resulting in $139 \mathrm{~T}$ peak centre axial field. Figure 9 shows the same coil with $1 \mathrm{~mm}$ thick SS lining along the inner wall, with same driving source parameters gave a peak field output of

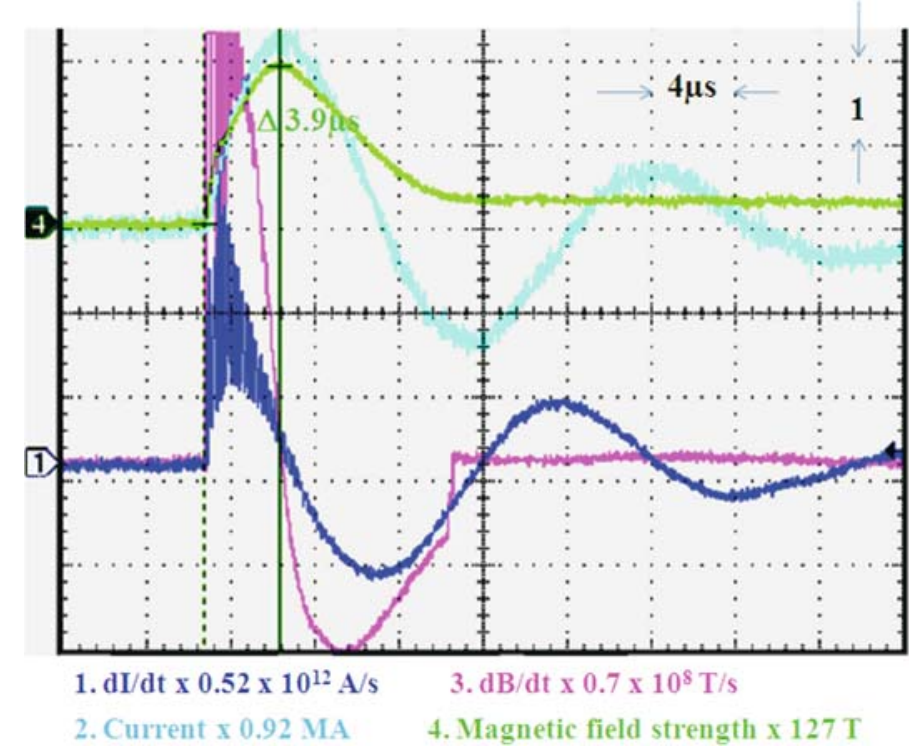

Figure 9. Experimental performance of an inertial magnet with $3.6 \mathrm{~mm}$ inner diameter, $3 \mathrm{~mm}$ long and $35 \mathrm{~mm}$ outer diameter with $1 \mathrm{~mm}$ thick SS lining. 
$255 \mathrm{~T}$. The magnetic field probe got destroyed at $3.6 \mu \mathrm{sec}$ and $11.7 \mu \mathrm{sec}$ without and with SS lining. The SS lining delays the damages to the inner wall and avoids the probe record loss due to the expanding metal vapors before peak magnetic field. The copper surrounding the SS lining traps the magnetic flux for longer time and results in long and high magnetic field pulse.

\section{Conclusions}

We have successfully generated magnetic field strength in excess of $257 \mathrm{~T}$ using the inertial magnet made out of a copper disk with a key hole of $3.6 \mathrm{~mm}$ diameter. Metal vapour interactions that render field record loss have been observed in high field shots, particularly above $200 \mathrm{~T}$ peak fields in $5 \mathrm{~mm}$ long coils and above $140 \mathrm{~T}$ peak fields in $3 \mathrm{~mm}$ long coils. The spatial non-uniform conductivity ( $\mathrm{SS}-\mathrm{Cu}$ coil) has enabled the complete field record in the case of $3 \mathrm{~mm}$ long coils. It is evident that magnetic field strength greater than $300 \mathrm{~T}$ can be generated with similar coils driven by MAGIC capacitor bank charged to $28 \mathrm{kV}$ and above. Efforts are underway to enhance the coil performance with input current sharpening and to measure the high magnetic field successfully without probe record loss using thin and strong armour around the B-dot probe.

We thank Shri L V Kulkarni, Shri A M Rawol and Shri A Patil for the technical help rendered in setting-up of the remote control and operation of the capacitor bank.

\section{References}

Frings Paul H, Luc Van Bockstal 1995 Strength versus conductivity. Physics B 211: 73-76

Furth H P, Waniek R W 1956 Production and use of high transient magnetic fields I. Rev. Sci. Instrum. 27(4): 195-203

Furth H P, Levine M A, Waniek R W 1957 Production and use of high transient magnetic fields II. Rev. Sci. Instrum. 28(11): 949-958

Knoepfel H 1970 Pulsed magnetic fields (Amsterdam and London: North Holland Publishing Company)

Miura N, Herlach F 1985 Pulsed strong and ultra strong magnetic fields-Applications of strong and ultra strong magnetic fields (Topics in Applied physics Vol. 57) (Heidelberg: Springer)

Portugall O, Puhlmann N, Muller H U, Barczewski M, Stolpe I, Von Ortenberg M 1999 Mega gauss magnetic field generation in Single-turn coils: New frontiers for scientific experiments. J. Phys. D: Appl. Phys. 32: 2354-2366

Shearer J W 1969 Interaction of the capacitor bank produced mega gauss fields with small single turn coils. J. Appl. Phys. 40(11): 4490-4497

Shneerson G A, Sergey I Krivosheev 2004 Development and achievements in single turn coil technique. Mega gauss $X$ Berlin, Germany

Singleton J 2004 The national high magnetic pulsed field facility at Los Alamos National Laboratory. Physica B 346: 614-617 